\begin{tabular}{|c|c|c|c|}
\hline BMI & $\mathbf{N}(\%)$ & SLEDAI Mean (SD) & $P$ value \\
\hline Low (reference) & $183(9.7)$ & $2.9(2.2)$ & REF \\
\hline Normal & $655(34.5)$ & $2.7(2.4)$ & 0.3107 \\
\hline Overweight & $531(28)$ & $2.5(2.1)$ & 0.0285 \\
\hline Obese & $290(15.3)$ & $2.6(2.1)$ & 0.0285 \\
\hline Severely Obese & $237(12.5)$ & $2.5(2.1)$ & 0.0587 \\
\hline
\end{tabular}

This analysis adds further support to the existence of an obesity paradox in SLE.

Funding Source(s): The Hopkins Lupus Cohort was funded by AR 69572

\section{STUDY ON SERUM DNAASE1 ACTIVITY IN PEDIATRIC ONSET SYSTEMIC LUPUS ERYTHEMATOSUS FROM A TERTIARY CARE CENTRE IN NORTH WEST INDIA}

${ }^{1}$ Nameirakpam Johnson*, ${ }^{2} \mathrm{~N}$ Ramalingeswara, ${ }^{3}$ Amit Rawat, ${ }^{4}$ Deepti Suri, ${ }^{4}$ Surjit Singh. ${ }^{1}$ Dept. of Pediatrics, Allergy- Immunology Unit, Postgraduate Institute of Medical Education and Research; ${ }^{2}$ Advanced Pediatrics Centre, Postgraduate Institute of Medical Education and Research; ${ }^{3} P G I M E R$ Chandigarh; ${ }^{4}$ Postgraduate Institute of Medical Education and Research, Chandigarh, India

\subsection{6/lupus-2019-Ism.278}

Background DNAase is an apoptotic endonuclease responsible for degradation of chromatin released by inappropriately cleared dead cells. DNAase1 activity in systemic lupus erythematosus (SLE) patients is lower than that in inactive disease in studies conducted in adult SLE patients from developed country. There is a paucity of data on DNAase1 activity in paediatric SLE from India.

Methods A cross-sectional observational study was conducted over a period of 1 year. Thirty-three consecutive children with pediatric-onset SLE were enrolled and divided into active and inactive disease activity groups based on Systemic Lupus Erythematosus Disease Activity Index (SLEDAI) score and compared the serum DNAase1 level between the two groups.

Results Out of 33 children enrolled, 13 (39.3\%) had active disease (SLEDAI score 3) and $20(60.6 \%)$ had inactive disease activity. Mean age at diagnosis was 8.5 years and 10.2 years in active and inactive groups respectively. There is female preponderance $(66.7 \%)$ in the enrolled patients. Anti nuclear antibody (ANA) was positive in $90.9 \%$ of patients. The most common pattern of ANA was diffuse pattern (48.4\%). The patients in active disease activity group presented most commonly with nephritis (53.8\%), rash (53.8\%), arthralgia (38.5\%), oral ulcer (30.8\%) and central nervous system (CNS) involvement (38.4\%) while patients in inactive disease activity group presented with nephritis (35\%), arthralgia (35\%), rash (25\%) and CNS involvement (30\%). Class III/IV lupus nephritis was present in $25 \%$ in active disease activity group while it was present in $23.1 \%$ in inactive disease activity group. Anti-double stranded DNA (anti dsDNA) was elevated in $53.8 \%$ in active group and $50 \%$ in inactive group. Antiphospholipid antibody was present in $3(23.1 \%)$ in active disease activity group and $5(25 \%)$ in inactive disease activity group. The mean serum concentrations of DNAase1 were $15.394 \mathrm{ng} /$ $\mathrm{ml}$ in active disease group and $15.205 \mathrm{ng} / \mathrm{ml}$ in inactive disease group. There was no statistically significant difference in the serum DNAase1 concentrations between the two groups $(p=0.943)$. There was also no significant difference in the mean serum concentration of DNAase1 in patients with or without nephritis $(p=0.080)$.

Conclusions The present study could not established any correlation between serum DNAase1 levels and disease activity in pediatric-onset SLE. There was no association between serum DNAase1 levels and organ involvement such as nephritis in the enrolled patients.

Funding Source(s): None

\section{SEXUAL DYSFUNCTION IN A BRAZILIAN COHORT OF BELIMUMAB TREATED SLE PATIENTS}

Morton Scheinberg*, Andrea Pimentel Fonseca Golmia, Ricardo Golmia, Gislene Ferrarezi, Aline Pinheiro dos Santos Cortada. Clinical Research Center Hospital AACD

\subsection{6/lupus-2019-Ism.279}

Background Systematic review and metaanalysis results of sexual dysfunction in patients with systemic lúpus erythematosus are contradictory. We investigate the quality of sexual activity in 74 patients with SLE receiving standard care of therapy and the addition of monthly injections of belimumab.

Methods The Female Sexual Function Index (FSfi), obtained by applying a 19-item questionnaire that assesses six domains (sexual desire, arousal, vaginal lubrication, orgasm, sexual satisfaction and pain), was used. 
Results The mean \pm SD was desire $162.9 \pm 10.02$ arousal 143.4 \pm 8.6 lubrification $213.7 \pm 13.4$ orgasm $214.4 \pm 14.8$ satisfaction $222.4 \pm 19.9$ pain $165.6 \pm 11.7$

Conclusions The FSFi in SLE patients are decreased when compared with what is reported in placebo populations but its magnitude is reduced when compared with results reported in other severe chronic diseases. This findings suggests that further studies are needed to find out if belimumab treated SLE patients have some degree of protective effect in sesxual dysfunction.

Funding Source(s): Funding source Institutional and GSK.

\section{COGNITIVE DYSFUNCTION IN AI-CTD IS ASSOCIATED WITH TRADITIONAL CARDIOVASCULAR RISK FACTORS BUT NOT IMMUNOLOGICAL PARAMETERS}

${ }^{1}$ Marta Aguilar Zamora*, ${ }^{2}$ Katie Dutton, ${ }^{2}$ Zoe Wigston, ${ }^{2}$ Adewonuola Alase, ${ }^{2}$ Antonios Psarras, ${ }^{2}$ Md Yuzaiful Md Yusof, ${ }^{2}$ Edward Vital. ${ }^{1}$ Hospital Universitario Dr. Peset, Valencia. Fundación Valenciana de Reumatologia; ${ }^{2}$ University of Leeds

\subsection{6/lupus-2019-Ism.280}

Background Cognitive dysfunction is a common problem in autoimmune connective tissue diseases such as SLE that can be screened for using the Montreal Cognitive Assessment (MoCA). The causes of cognitive dysfunction are poorly understood. They may include immune-mediated neurological dysfunction (which should be targeted using immunosuppression) or traditional cardiovascular risk factors (which may be treated as in non-SLE patients). The purpose of this study was to explore these counter-hypotheses in patients with established autoimmune disease by analysing conventional measures of autoimmunity, cardiovascular risk, as well as validated scores for interferon status. Since cumulative organ damage and toxicity of therapy may affect these patients, we also included a cohort of At Risk individuals as previously described. ${ }^{1}$

Methods We assessed three cohorts: (1) patients with established AI-CTD (SLE, Sjogrens syndrome or undifferentiated CTD >12 months); (2) at risk individuals referred to secondary care due to ANA +and symptoms suggestive of AI-CTD less than 12 months duration; (3) age and sex matched healthy controls. Cognitive dysfunction was tested using the MoCA. Cardiovascular risk was assessed by recording diabetes, hypertension, previous angina or AMI, atrial fibrillation and cholesterol. Type I interferon activity was assessed using a validated two-score system for IFN status previously described. $^{2}$

Results As expected, the number of patients with an abnormal MOCA score was greater in AI-CTD and At-Risk individuals than in the healthy controls (HC: 20\%; At Risk: 39\%; SLE: $34 \%)$. Also as expected, the IFN scores varied significantly between these groups $(\mathrm{p}=0.046, \mathrm{~F}=4.66)$.

We compared parameters between individuals with normal and abnormal MoCA scores within each group and in all groups. Results are shown in table 1 .

In patients with an AI-CTD, the cognitive function assessed wasnt associated to any of the immune-related but associated

Abstract 280 Table 1 Results

\begin{tabular}{|c|c|c|c|c|c|c|c|c|c|}
\hline \multirow[t]{2}{*}{ Parameter } & \multicolumn{3}{|c|}{ At Risk } & \multicolumn{3}{|c|}{ Al-CTD } & \multicolumn{3}{|c|}{ All patients } \\
\hline & No $C D(n=17)$ & $\mathrm{CD}(\mathrm{n}=11)$ & $\mathrm{p}$ & No $C D(n=21)$ & $C D(n=11)$ & $\mathrm{p}$ & No $C D(n=50)$ & $C D(n=25)$ & $\mathrm{p}$ \\
\hline Age & $\begin{array}{c}58.47 \text { (SD } \\
12.41)\end{array}$ & 58.09 (SD17.81) & ns & 44.5 (SD 12.71) & 55 (SD 14.71) & 0.06 & $\begin{array}{c}50,98 \text { (SD } \\
15.17)\end{array}$ & $\begin{array}{c}55.96 \text { (SD } \\
15.13)\end{array}$ & ns \\
\hline Gender (female) & $94 \%$ & $63 \%$ & 0.04 & $73 \%$ & $70 \%$ & $\mathrm{~ns}$ & $83 \%$ & $70 \%$ & ns \\
\hline $1 \mathrm{CV}$ risk factor & $35 \%$ & $54 \%$ & ns & $19 \%$ & $54 \%$ & 0.04 & $32 \%$ & $52 \%$ & 0.06 \\
\hline Fibromyalgia & $17.6 \%$ & $18 \%$ & ns & $14.3 \%$ & $0 \%$ & $\mathrm{~ns}$ & $20.4 \%$ & $8 \%$ & $\mathrm{~ns}$ \\
\hline Neuropsychiatric Manifestations & $11.7 \%$ & $9 \%$ & $\mathrm{~ns}$ & $5 \%$ & $0 \%$ & ns & $8 \%$ & $4.5 \%$ & ns \\
\hline Current glucocorticoids & $0 \%$ & $0 \%$ & ns & $23.8 \%$ & $9 \%$ & ns & $13 \%$ & $4.5 \%$ & ns \\
\hline Smoking & $12.5 \%$ & $12.5 \%$ & $\mathrm{~ns}$ & $25 \%$ & $9 \%$ & $\mathrm{~ns}$ & $16.2 \%$ & $9 \%$ & ns \\
\hline $\lg G$ & 11.3 (SD 2.29) & 13 (SD 2.47) & ns & 13.65 (SD 3.88) & 14.42 (SD 8.94) & ns & 12.47 (SD 3.46) & 13.55 (SD 5.91) & ns \\
\hline ESR & $8(S D 2.64)$ & - & - & $\begin{array}{c}20.43(\mathrm{SD} \\
21.47)\end{array}$ & 11 (SD 8.44) & ns & 16.70 & 11 & ns \\
\hline C3 & 1.29 (SD 0.33) & 1.37 (SD 0.18) & $\mathrm{ns}$ & 1.31 (SD 0.34) & 1.24 (SD 0.49) & ns & $1.30(\mathrm{SD} 0.33)$ & $1.32(S D 0.33)$ & ns \\
\hline C4 & 0.24 (SD 0.09) & 0.25 (SD 0.06) & ns & 0.25 (SD 0.11) & 0.26 (SD 0.1) & $\mathrm{ns}$ & 0.25 (SD 0.1) & 0.25 (SD 0.07 & $\mathrm{~ns}$ \\
\hline Number of ENA & 1.17 (SD 0.72) & 1.27 (SD 0.9) & Ns & 1.66 (SD 1.74) & 1.27 (SD 1.27) & $\mathrm{ns}$ & 1.44 (SD 1.39) & 1.27 (SD 1.07) & ns \\
\hline Physician VAS & 0.38 (SD 0.66) & 1.05 (SD 1) & $\mathrm{ns}$ & 2.25 (SD 2.06) & 1.72 (SD 1.11) & ns & 1.37 (SD 1.81) & 1.37 (SD 1.08) & ns \\
\hline Patient VAS & 3.5 (SD 2.5) & 4.85 (SD 3.02) & $\mathrm{ns}$ & 4.17 (SD 2.5) & 4.6 (SD 3.1) & ns & 3.83 (SD 2.43) & 4.80 (SD 2.89) & ns \\
\hline Education $<12 y$ & $20 \%$ & $36 \%$ & $\mathrm{~ns}$ & $17.6 \%$ & $50 \%$ & $\mathrm{~ns}$ & $13.6 \%$ & $37.5 \%$ & 0.023 \\
\hline Currently employed & $21.4 \%$ & $20 \%$ & ns & $85 \%$ & $40 \%$ & 0.019 & $53 \%$ & $28.5 \%$ & ns \\
\hline Anxiety or depression & $33.3 \%$ & $72.7 \%$ & 0.047 & $60 \%$ & $50 \%$ & $\mathrm{~ns}$ & $57.5 \%$ & $58.3 \%$ & ns \\
\hline IFN Score A & 6.23 (SD 1) & 5.48 (SD 1.26) & $\mathrm{ns}$ & 5.22 (SD 1.22) & 5.15 (SD 1.95) & ns & 5.64 (SD 1.22) & 5.32 (SD 1.61) & ns \\
\hline IFN Score B & 6.49 (SD 0.9) & 5.87 (SD 0.89) & $\mathrm{ns}$ & 5.7 (SD 1.15) & 6.27 (SD 1.46) & ns & 6.02 (SD 1.11) & 6.07 (SD 1.2) & ns \\
\hline
\end{tabular}

